\title{
Ferroelectric Surfaces for Cell Release
}

Henrik Toss, Susanna Lönnqvist, David Nilsson, Anurak Sawatdee, Josefin Nissa, Simone

Fabiano, Magnus Berggren, Gunnar Kratz and Daniel T Simon

\author{
Journal Article
}

\section{Tweet}

N.B.: When citing this work, cite the original article.

Original Publication:

Henrik Toss, Susanna Lönnqvist, David Nilsson, Anurak Sawatdee, Josefin Nissa, Simone Fabiano, Magnus Berggren, Gunnar Kratz and Daniel T Simon, Ferroelectric Surfaces for Cell Release, Synthetic metals, 2017. 228, pp.99-104.

http://dx.doi.org/10.1016/j.synthmet.2017.04.013

Copyright: Elsevier

http://www.elsevier.com/

Postprint available at: Linköping University Electronic Press

http://urn.kb.se/resolve?urn=urn:nbn:se:liu:diva-121804

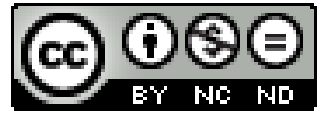




\title{
Ferroelectric Surfaces for Cell Release
}

\author{
Henrik Toss ${ }^{1}$, Susanna Lönnqvist ${ }^{2}$, David Nilsson ${ }^{3}$, Anurak Sawatdee ${ }^{3}$, Josefin Nissa ${ }^{1}$, Simone Fabiano ${ }^{1}$, \\ Magnus Berggren ${ }^{1}$, Gunnar Kratz ${ }^{2,4}$, Daniel T. Simon ${ }^{1 *}$ \\ 1. Laboratory of Organic Electronics, Department of Science and Technology, Linköping University, SE- \\ 60174 Norrköping, Sweden \\ 2. Department of Clinical and Experimental Medicine, Linköping University, SE-581 83 Linköping, Sweden \\ 3. Acreo Swedish ICT AB, SE-601 17 Norrköping, Sweden \\ 4. Department of Hand and Plastic Surgery, Linköping University, SE- 58183 Linköping, Sweden \\ * Corresponding author: daniel.simon@liu.se
}

Adherent cells cultured in vitro must usually, at some point, be detached from the culture substrate. Presently, the most common method of achieving detachment is through enzymatic treatment which breaks the adhesion points of the cells to the surface. This comes with the drawback of deteriorating the function and viability of the cells. Other methods that have previously been proposed include detachment of the cell substrate itself, which risks contaminating the cell sample, and changing the surface energy of the substrate through thermal changes, which yields low spatial resolution and risks damaging the cells if they are sensitive to temperature changes. Here cell culture substrates, based on thin films of the ferroelectric polyvinylidene fluoride trifluoroethylene (PVDF-TrFE) co-polymer, are developed for electroactive control of cell adhesion and enzyme-free detachment of cells. Fibroblasts cultured on the substrates are detached through changing the direction of polarization of the ferroelectric substrate. The method does not affect subsequent adhesion and viability of reseeded cells.

\section{Introduction}

When culturing adherent cells for purposes ranging from basic research to regenerative medicine, the cells must at some point be detached from their culture substrate. Today, proteolytic treatment with trypsin is one of the most widely used methods to achieve this detachment, and is typical of all biochemical detachment techniques. Through trypsinization, the protease cleaves cell adhesion proteins such as integrins at the carboxylic side of the amino acids arginine and lysine. [1] For sub-cultured cells, this cleavage of integrins in the plasma membrane leads to desired detachment of adherent cells by the loss of cell-tocell and cell-to-substrate interactions. However, the treatment will also affect several other plasma membrane proteins, which typically deteriorates the quality and function of the cultured cells. Specifically, growth factor receptors, cadherins and integrins in the plasma membrane play crucial roles as mitogen receptors, anchoring proteins and signal effectors for migration and adhesion, and trypsinization can lead to altered function and lower viability of cells. Even after up to 24 hours of recovery times following a trypsin treatment, whole proteome changes have been observed [2] and significant loss of integrin expression has been measured. Furthermore, high trypsin concentrations can alter the formation and number of focal adhesions, the anchoring complex forming connections between the extracellular matrix and the cytoplasmic part of integrins, with profound effects on cell spreading and adhesion. [3]

One application where viability and integrin integrity of detached cells is crucial is the treatment of burn wounds. The introduction of an enzyme-free detachment technique for cells and cell sheets, in a customized cell culture system, could address problems faced with basically all current burn treatment strategies based on cell cultures. The foremost method for treatment of large burns is the use of skin autografts (i.e., skin taken from elsewhere on the patient's body), but in the case of extensive burns, donor sites on the patient are limited and donor-site morbidity is high. [4] Epithelial autografts expanded and cultured in vitro provide an alternative to traditional skin grafts, but the method is hampered by the long cultivation times that are required, high costs and sensitivity to infection. [5] The method has come to be viewed as an early closure tool but not suitable as permanent coverage due to occurrence of blistering, hyperkeratosis and scar contraction. [4] Transplantation of pre-confluent 
epithelial cells in suspension has been a way to address the issues associated with full epithelial autografts. [6] The size of the required donor site is substantially reduced and the culture times in vitro are significantly shortened. [4] Further, the use of cultured epithelial cells provide a desired proliferative cell population and is thus advantageous for treatment of burns. [7] Unfortunately, trypsinization of these immediately prior to transplantation can impair their binding ability and possibly reduce overall viability. An enzyme-free detachment technique could yield high quality, fully viable cells for transplantation in suspension. Considering cultured autografts, an addressable cell culture dish intended for expansion, culture, transport and detachment of the autograft could also reduce the labor-intensity of preparing the grafts and facilitate a handling practice that lowers the risk of infection.

Strategies toward active enzyme-free cell release with spatiotemporal control have previously been explored and applied, such as electroactive release of the substrate surface [8-11] and cell release triggered by thermally induced changes of the surface properties of the substrate. [12-14] It is typically difficult to achieve high spatial control with a thermal strategy due to thermal conductivity through the substrate. Temperature changes could also affect the function and viability of the cells. [15] Electroactive release can provide high spatiotemporal resolution of the cell detachment, offering the possibility of choosing which cell(s) to release and when to release them. Similar to other demonstrations of electroactivelytriggered cell detachment[8-10], our previous approaches were based on release of a layer of the substrate material along with the cells. [11] While effective at non-enzymatic cell detachment, this technique could result in contamination of the resulting graft. Furthermore, the electroactive surface is not reversible: the release layer is dispersed along with the released cells and cannot be recovered. Cell detachment via electroactive control over surface properties, without release of the substrate material, is thus an appealing idea, providing a detachment technique with minimal risk of contamination from the substrate itself.

Ferroelectric surfaces, with defined polarization patterns, have been proposed as substrates to guide the growth patterns of cell cultures. [1618]These surfaces provide a well-defined surface charge when polarized in an aqueous environment as the charge is fixed on the surface, resulting in non-faradaic charging. $[19,20]$ In an electrode/ferroelectric film/aqueous electrolyte/electrode configuration (Figure 1), the state of the ferroelectric film can undergo a phase transition upon electric biasing. [21] This yields in situ control of the surface properties - specifically the surface charge, and thus the adherence of cells - at high spatiotemporal resolution and without any contamination of the detached cells. [16] We thus propose the possibility of inducing cell release through changing the surface properties of a cell substrate including a ferroelectric thin film as the outermost, cell-facing layer. The surface charge is changed by reversing the polarization vector in the ferroelectric polymer thin film, biased through an electrolyte constituted by the cell culture medium, using short voltage pulses. For the ferroelectric polymer film, we have explored the polyvinylidene fluoride trifluoroethylene (PVDF-TrFE) co-polymer.

\section{Results}

\subsection{Ferroelectric characterization}

The ferroelectric cell release device is based on an electrode/ferroelectric film/aqueous electrolyte/electrode configuration (Figure 1). Specifically, the device consists of a thin film $(\sim 140 \mathrm{~nm})$ of the ferroelectric PVDF-TrFE copolymer with a monomer weight ratio of 70/30 PVDF-TrFE coated over an indium tin oxide (ITO, $15-30 \mathrm{~nm}$ ) bottom electrode on a glass carrier substrate $(25 \times 25 \times 1.1 \mathrm{~mm})$. A polypropylene ring (15 mm diameter) was glued on the PVDFTrFE surface, forming an ad hoc Petri dish with a ferroelectric bottom surface. For ferroelectric characterization, this liquid well was filled with phosphate buffered saline (PBS), and a coiled $\mathrm{Al}$ wire counter electrode (to maximize surface area) was immersed in the PBS.

Voltage was applied between the ITO bottom electrode and $\mathrm{Al}$ counter electrode as shown in Figure 1A. A full characterization switch cycle was established by incrementing the voltage from $0 \mathrm{~V}$ to $18 \mathrm{~V}$, then to $-18 \mathrm{~V}$ and finally back to $0 \mathrm{~V}$, with each voltage point consisting of $5 \mathrm{~ms}$ voltage pulse followed by a 500 ms delay. A typical polarization curve of such device setup is given in Figure 1B. By sweeping the voltage from zero or a negative voltage to positive voltages, a current peak appears at around $5 \mathrm{~V}$. When reversing the sweep a negative current peak is observed at an opposite polarity of $-5 \mathrm{~V}$. It is important to note that upon programming the ferroelectric layer with a voltage sequence of the same polarity no current peak is visible during the second sweep corroborating the fact that ion migration is not the origin of the capacitance bistability (see Figure 1B). Upon applying a voltage signal to the 
electrodes, the ions of the electrolyte will form dense Helmholtz layers along the surface of the Al-electrode and the top surface of the PVDFTrFE film. We have recently shown that electrolytes can provide enough of charges to compensate the surface dipoles of a ferroelectric thin film (and vice versa). Furthermore, this process is not limited by the polarization switching time of the Helmholtz (electric double) layer nor of the ferroelectric layer. [19] A simplified equivalent circuit would represent the Helmholtz layers at the two electrodes as capacitors connected in series. The electrically insulating ferroelectric film will make the separation of charges, in the Helmholtz layer at that surface, larger. In practice this means that the capacitance of the Helmholtz layer at the polymer film will be the smaller of the two and the majority of the voltage drop will thus be over this capacitor, i.e., the ferroelectric polymer film. The polarization switching of the PVDF-TrFE film occurred around $\pm 5 \mathrm{~V}$ and the polarization was found to be remnant and stable over extended periods of time. The PVDF-TrFE films were around $140 \mathrm{~nm}$ thick, so the corresponding coercive field was $\sim 36 \mathrm{MV} / \mathrm{m}$. At $\pm 15 \mathrm{~V}$ the current was very low and we can thus assume that the entirety of the film was polarized at the voltages around $\pm 5 \mathrm{~V}$ (see Figure 1B). Hence, the surface charge density of PVDF-TrFE can be induced to be either positive or negative depending on the direction of the applied polarizing electric field. At positive voltage above $5 \mathrm{~V}$ applied to the ITO-electrode, the ferroelectric dipoles of the PVDF-TrFE film were aligned toward the aqueous electrolyte, expressing a net positive surface charge density (Figure 1C). By flipping the dipoles of the PVDF-TrFE film, the surface charge exposed to the electrolyte alters (i.e. negative surface charge density), potentially affecting the binding characteristics of extracellular proteins to the surface.

\subsection{Cell adhesion and viability}

To investigate the cell detachment characteristics from the ferroelectric PVDF-TrFE we chose fibroblasts as the model system. Fibroblasts are stromal cells responsible for the structural integrity of connective tissues, and are principal producers of its components such as collagen. In cutaneous wound healing fibroblasts play a crucial role as they produce the granulation tissue and highly interact with epidermal keratinocytes in the proliferative phase of the wound healing process. [22] Human primary fibroblasts were seeded on polarized and unpolarized PVDF-TrFE surfaces and no difference in morphology could be observed (Figure S1). We then determined the passive characteristics of the PVDF-TrFE surface by assessing fibroblast viability with an MTTassay on the pristine ferroelectric surfaces, compared to standard cell culture polystyrene surfaces. In the MTT-assay, the MTT dye is enzymatically reduced to formazan by viable cells only, and the absorbance of the formazan is thus a metric for overall cell viability. Compared to viability on the polystyrene control surfaces (defining $100 \%$ viability), the fibroblasts exhibited significantly lower viability at 24 hours (82.0\% \pm 6.5 of control viability) and at 48 hours (83.2\% \pm 3.4 of control viability) after seeding on PVDF-TrFE (Figure 2A). As the MTT-assay reflects the number of viable cells in the samples with equal seeding concentrations, the findings on viability at 24 hours indicate that approximately $20 \%$ of seeded cells failed to attach, compared to cell culture polystyrene. We investigated attachment of fibroblasts to the unpolarised PVDF-TrFE surfaces. Rapid adhesion was achieved by $33.4 \%$ of seeded fibroblasts within 15 minutes, by 30 minutes $60.5 \%$ and by 60 minutes $65.8 \%$ of the seeded cells were attached to the unpolarized surface (Figure 2B). The morphology of the primary fibroblasts on unpolarized PVDF-TrFE is similar (Figure 2C) to that of fibroblasts on cell culture polystyrene (Figure 2D).

\subsection{Cell release}

We proceeded to evaluate cell detachment performance by culturing primary fibroblasts for 24 hours on pristine PVDF-TrFE surfaces in cell medium. The film was subsequently polarized by applying $16 \mathrm{~ms}$ pulses of either positive or negative $15 \mathrm{~V}$. From current versus voltage characterization, we found that the PVDF-TrFE films did not fully switch after the application of only one or two $16 \mathrm{~ms}$ pulses, but did fully polarize with three subsequent pulses. The application of three successive $-15 \mathrm{~V}$ pulses (ITO as cathode) to pristine, unpolarized surfaces yielding negative charge on the PVDF-TrFE surface facing the cells - resulted in partial detachment of fibroblast cells (Figure 3B, ii-iii). Additional $-15 \mathrm{~V}$ pulsing resulted in further fibroblast detachment (Figure 3B, iv). Analogous positive voltage pulsing (ITO as anode), yielding positive charge on the PVDF-TrFE surface facing the cells, did not affect cell detachment as strongly as negative voltage pulsing. However, after detachment, cells were collected and re-seeded on cell culture polystyrene and displayed normal morphology after adhesion (Figure 3B, v; Figure S2). 


\section{Discussion}

While the results above indicate successful ferroelectric cell release, the electrical characteristics of the experiment deviate from ideal ferroelectric thin film behavior. Firstly, the exact location of the current peaks is not consistent, and there are often smaller peaks occurring at a somewhat lower voltage, as well as some remaining current at higher voltages. This could be due to variation in the film thickness or the size of the polymer crystals in the film. Cleaner, more ideal thin film behavior could likely be achieved using thicker films, yielding a smoother Gaussian-like current peak by providing a wider distribution of crystal dimensions. Thicker films would necessitate higher voltages to achieve remnant polarization of the entire film. However, the current levels would be unaffected by the film thickness and as the largest part of the potential drop would be over the electrically insulating ferroelectric film. Indeed, a recent study of the ferroelectric-electrolyte interface indicates minimal electric fields at the liquid interface.[21] Thus, increased film thickness and applied voltage should have little effect on the viability of the cells.

In this initial demonstration of ferroelectric cell release, we did not investigate the specific mechanism of cell detachment at the biochemical level. Fibroblasts themselves have a net negative surface charge [23], so static repulsion could be a factor of the release mechanism. Indeed, previous studies have shown that fibroblasts preferentially adhere to positively charged surfaces. [24] Seeding of primary fibroblasts on polarized and pristine PVDF-TrFE substrates revealed no difference in morphology of the human primary fibroblasts in accordance with findings of Christofis et al. [18] where rat embryonic fibroblasts did not show preference of either polarization of a $\mathrm{LiTaO}_{3}$ substrate in a side by side comparison on a patterned surface, but the cells avoided polarization gradients between the polarization domains. Some negatively charged polymer surfaces have been shown to negatively affect cell adhesion [25] and cell binding proteins. [26] Regarding binding proteins, differences in their conformation on pure PVDF surfaces between the poled and unpoled states of the ferroelectric film have previously been reported. [27] The proteins on the surface may also undergo a conformational reorganization upon a changed polarization state of the surface. During this process, the number of attachment points, both to the surface and the cells, would temporarily change and lead to a looser attachment of the cells.
With a canonical ferroelectric film, applying additional pulses to an already-polarized surface would not affect the film. However, we observed some additional cell release with such additional pulses. This effect could be due to the small, purely dielectric charge induced in the PVDFTrFE film that is not remnant, yet still present when further voltage is applied. The change in surface charge due to the dielectric properties is very small compared to the charge caused by ferroelectric polarization. A $15 \mathrm{~V}$ pulse would induce a dielectric surface charge of approx. $7 \mathrm{mCm}^{-2}$, compared to the change in surface charge density due to ferroelectric polarization of 2*100 $\mathrm{mCm}^{-2}$ when switching from one polarity to the other. However, this $7 \mathrm{mCm}^{-2}$ may be enough to prevent the already-destabilized cells from settling, increasing their likelihood to detach completely.

To develop full, cultured epithelial autografts for burn and wound healing, both fibroblasts (required for the extracellular matrix) and keratinocytes (epidermal cells) are required. In this study, we demonstrated fibroblast release, and attempted keratinocyte detachment. However, we did not succeed in releasing this polar, strictly sheet forming cell type. Keratinocytes would be of the highest interest for transplantation purposes for burn wounds and chronic wound treatment as they are the cell type responsible for the reepithelialization of a wound. However, the interplay between keratinocytes and fibroblasts in wound healing is intricate and drives the progression of the wound healing process from an inflammatory stage towards a highly proliferative phase, which enables keratinocyte migration and re-epithelialization of the wound. Transplantation of both - or either - cell types could be beneficial as inclusion of the native components, with all surface proteins intact as in the case of ferroelectric release, would support the normal reciprocity of the two cell types in the skin. Cell sheet engineering could highly benefit from a cell culture dish with an integrated release mechanism, as cultured cell sheets are fragile and difficult to manage. The handling of cell sheets and transport from cell culture to the operating room would be simplified and made safer, as the cell sheets would not be required to be handled prior to the detachment of the sheet at the time of transplantation.

PVDF and PVDF-TrFE co-polymers have in the past been shown to have good biocompatibility [28] and have been used as materials in implantable devices and to steer stem cell fate. [29-31] Furthermore, a variety of devices and 
components, such as ferroelectric memories, have previously utilized the local polarization of ferroelectric films. [32] Combining these precedents with the cell release described above, one can easily envision local cell release in commercial biotechnology tools via local polarization, for example by placing the ferroelectric film on an addressable matrix. In this manner, high spatial resolution of selective cell release could be achieved in which only some areas are polarized, releasing only the cells - or even single cell - on that specific area.

\section{Conclusions}

We have demonstrated that PVDF-TrFe surfaces are suitable as cell culture substrates for human dermal fibroblasts and that the polarity of the film affects the adhesion of cells to the culture surface. The polarity of the PVDF-TrFE film can be addressed with cells present on the surface and with the cells and ferroelectric surfaced immersed in culture medium, and the switching of the dipoles in the film affects the adhesion of cultured fibroblasts. Detachment of fibroblasts in this manner does not affect their subsequent adhesion or morphology. Ferroelectric cell release thus provides a promising new route to completely non-destructive, electronically addressable cell detachment.

\section{Supporting Information}

Materials and methods are detailed in the Supporting Information.

\section{Acknowledgements}

This work was carried out within the Organic Bioelectronics (OBOE) research environment (www.oboecenter.se), funded by a grant from the Swedish Governmental Agency for Innovation Systems (VINNOVA, 2010-00507). Additional support was provided by the Knut and Alice Wallenberg Foundation and the Önnesjö Foundation. The authors would also like to thank Jonathan Rakar for all the help with various software related issues. $\mathrm{H}$. Toss and S. Lönnqvist contributed equally to this work.

[1] J.V. Olsen, S.-E. Ong, M. Mann, Trypsin Cleaves Exclusively C-terminal to Arginine and Lysine Residues, Molecular \& Cellular Proteomics. 3 (2004) 608-614. doi:10.1074/mcp.T400003MCP200.

[2] H.L. Huang, H.W. Hsing, T.C. Lai, Y.W. Chen, T.R. Lee, H.T. Chan, et al., Trypsin-induced proteome alteration during cell subculture in mammalian cells, J Biomed Sci. 17 (2010) 36. doi:10.1186/14230127-17-36.
[3] M.A. Brown, C.S. Wallace, C.C. Anamelechi, E. Clermont, W.M. Reichert, G.A. Truskey, The use of mild trypsinization conditions in the detachment of endothelial cells to promote subsequent endothelialization on synthetic surfaces, Biomaterials. 28 (2007) 3928-3935.

[4] J.N. McHeik, C. Barrault, G. Levard, F. Morel, F.X. Bernard, J.C. Lecron, Epidermal healing in burns: autologous keratinocyte transplantation as a standard procedure: update and perspective, Plast Reconstr Surg Glob Open. 2 (2014) e218. doi:10.1097/GOX.0000000000000176.

[5] J.E. Paddle-Ledinek, D.G. Cruickshank, J.P. Masterton, Skin replacement by cultured keratinocyte grafts: an Australian experience, Burns. 23 (1997) 204-211.

[6] F.H. Gage, Cell therapy, Nature. 392 (1998) 18-24.

[7] J.C. Adams, F.M. Watt, Changes in keratinocyte adhesion during terminal differentiation: reduction in fibronectin binding precedes alpha 5 beta 1 integrin loss from the cell surface, Cell. 63 (1990) 425-435.

[8] O. Guillaume-Gentil, Y. Akiyama, M. Schuler, C. Tang, M. Textor, M. Yamato, et al., Polyelectrolyte Coatings with a Potential for Electronic Control and Cell Sheet Engineering, Adv. Mater. 20 (2008) 560565. doi:10.1002/adma.200700758.

[9] R. Inaba, A. Khademhosseini, H. Suzuki, J. Fukuda, Electrochemical desorption of self-assembled monolayers for engineering cellular tissues, Biomaterials. $30 \quad$ (2009) 3573-3579. doi:10.1016/j.biomaterials.2009.03.045.

[10] X. Jiang, R. Ferrigno, M. Mrksich, G.M. Whitesides, Electrochemical Desorption of Self-Assembled Monolayers Noninvasively Releases Patterned Cells from Geometrical Confinements, Journal of the American Chemical Society. 125 (2003) 23662367. doi:10.1021/ja029485c.

[11] K.M. Persson, R. Karlsson, K. Svennersten, S. Löffler, E.W.H. Jager, A. Richter-Dahlfors, et al., Electronic Control of Cell Detachment Using a SelfDoped Conducting Polymer, Adv. Mater. 23 (2011) 4403-4408. doi:10.1002/adma.201101724.

[12] H. Hatakeyama, A. Kikuchi, M. Yamato, T. Okano, Patterned biofunctional designs of thermoresponsive surfaces for spatiotemporally controlled cell adhesion, growth, and thermally induced detachment, Biomaterials. 28 (2007) 3632-3643. doi:10.1016/j.biomaterials.2007.04.019.

[13] J. Yang, M. Yamato, C. Kohno, A. Nishimoto, H. Sekine, F. Fukai, et al., Cell sheet engineering: Recreating tissues without biodegradable scaffolds, Biomaterials. $26 \quad$ (2005) 6415-6422. doi:10.1016/j.biomaterials.2005.04.061.

[14] T. Okano, N. Yamada, M. Okuhara, H. Sakai, Y. Sakurai, Mechanism of cell detachment from temperature-modulated, hydrophilic-hydrophobic polymer surfaces, Biomaterials. 16 (1995) 297-303. doi:10.1016/0142-9612(95)93257-E.

[15] A.J. McGahon, S.J. Martin, R.P. Bissonnette, A. Mahboubi, Y. Shi, R.J. Mogil, et al., The End of the (Cell) Line: Methods for the Study of Apoptosis in Vitro, Methods in Cell Biology. 46 (1995) 153-185. doi:10.1016/S0091-679X(08)61929-9.

[16] R. Ferris, B. Yellen, S. Zauscher, Ferroelectric Thin Films in Fluidic Environments: A New Interface for Sensing and Manipulation of Matter, Small. 8 (2012) 28-35. doi:10.1002/smll.201101173. 
[17] V. Marchesano, O. Gennari, L. Mecozzi, S. Grilli, Pietro Ferraro, Effects of Lithium Niobate Polarization on Cell Adhesion and Morphology, ACS Appl. Mater. Interfaces. 7 (2015) 1811318119. doi:10.1021/acsami.5b05340.

[18] C. Christophis, E. Cavalcanti-Adam, M. Hanke, K. Kitamura, A. Gruverman, M. Grunze, et al., Adherent cells avoid polarization gradients on periodically poled $\mathrm{LiTaO} 3$ ferroelectrics, Biointerphases. 8 (2013) 27. doi:10.1186/15594106-8-27.

[19] S. Fabiano, X. Crispin, M. Berggren, Ferroelectric Polarization Induces Electric Double Layer Bistability in Electrolyte-Gated Field-Effect Transistors, ACS Appl. Mater. Interfaces. 6 (2014) 438-442. doi:10.1021/am404494h.

[20] R.J. Ferris, S. Lin, M. Therezien, B.B. Yellen, S. Zauscher, Electric Double Layer Formed by Polarized Ferroelectric Thin Films, ACS Appl. Mater. Interfaces. 5 (2013) 2610-2617. doi:10.1021/am3031954.

[21] H. Toss, N. Sani, S. Fabiano, D.T. Simon, R. Forchheimer, M. Berggren, Polarization of ferroelectric films through electrolyte, Journal of Physics: Condensed Matter. 28 (2015) 105901. doi:10.1088/0953-8984/28/10/105901.

[22] S. Werner, T. Krieg, H. Smola, Keratinocytefibroblast interactions in wound healing, J Invest Dermatol. $\quad 127 \quad$ (2007) 998-1008. doi:10.1038/sj.jid.5700786.

[23] K. Yamamoto, M. Yamamoto, H. Ooka, Changes in negative surface charge of human diploid fibroblasts, TIG-1, during in vitro aging, Mech. Ageing Dev. 42 (1988) 183-195.

[24] M. Hamdan, L. Blanco, A. Khraisat, I.F. Tresguerres, Influence of Titanium Surface Charge on Fibroblast Adhesion, Clinical Implant Dentistry and Related Research. 8 (2006) 32-38. doi:10.2310/j.6480.2005.00028.x.

[25] P.B. van Wachem, A.H. Hogt, T. Beugeling, J.
Feijen, A. Bantjes, J.P. Detmers, et al., Adhesion of cultured human endothelial cells onto methacrylate polymers with varying surface wettability and charge, Biomaterials. 8 (1987) 323-328. doi:10.1016/0142-9612(87)90001-9.

[26] G.F. Khan, W. Wernet, Adsorption of proteins on electro-conductive polymer films, Thin Solid Films. 300 (1997) 265-271. doi:10.1016/S00406090(96)09518-1.

[27] C. Ribeiro, J.A. Panadero, V. Sencadas, S. LancerosMéndez, M.N. Tamaño, D. Moratal, et al., Fibronectin adsorption and cell response on electroactive poly(vinylidene fluoride) films, Biomedical Materials. 7 (2012) 035004.

[28] P. Hitscherich, S. Wu, R. Gordan, L.H. Xie, T. Arinzeh, E.J. Lee, The effect of PVDF-TrFE scaffolds on stem cell derived cardiovascular cells, Biotechnol. Bioeng. 113 (2016) 1577-1585. doi:10.1002/bit.25918.

[29] G. Laroche, Y. Marois, R. Guidoin, M.W. King, L. Martin, T. How, et al., Polyvinylidene fluoride (PVDF) as a biomaterial: from polymeric raw material to monofilament vascular suture, J. Biomed. Mater. Res. 29 (1995) 1525-1536. doi:10.1002/jbm.820291209.

[30] T. Okoshi, H. Chen, G. Soldani, P.M. Galletti, M. Goddard, Microporous small diameter PVDF-TrFE vascular grafts fabricated by a spray phase inversion technique, Asaio J. 38 (1992) M201-6.

[31] J. Li, X. Mou, J. Qiu, S. Wang, D. Wang, D. Sun, et al., Surface Charge Regulation of Osteogenic Differentiation of Mesenchymal Stem Cell on Polarized Ferroelectric Crystal Substrate, Adv. Healthcare Mater. 4 (2015) 998-1003. doi:10.1002/adhm.201500032.

[32] R.C. Naber, K. Asadi, P.W. Blom, D.M. de Leeuw, B. de Boer, Organic nonvolatile memory devices based on ferroelectricity, Adv. Mater. 22 (2010) 933-945. 


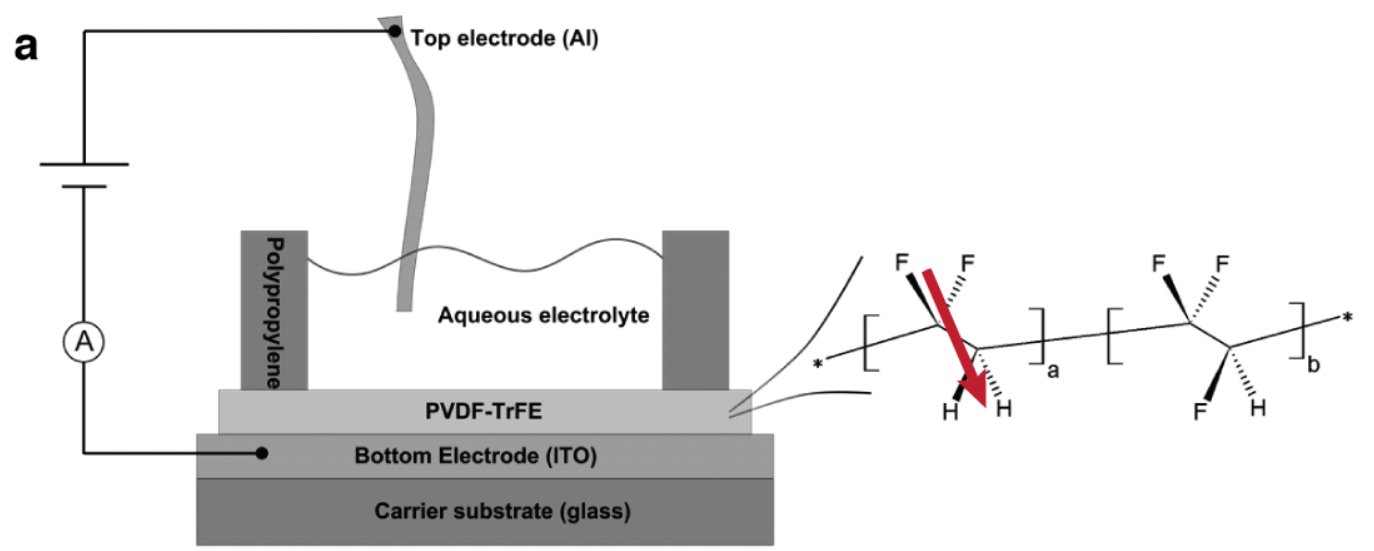

b

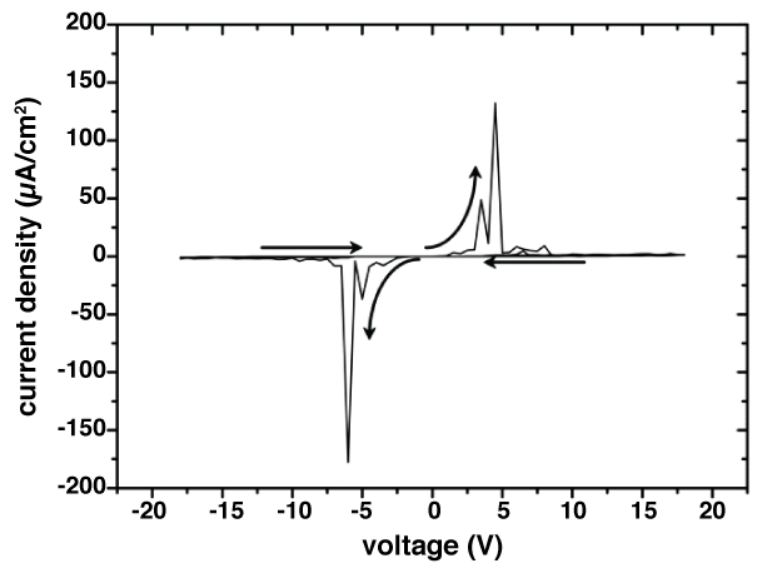

c

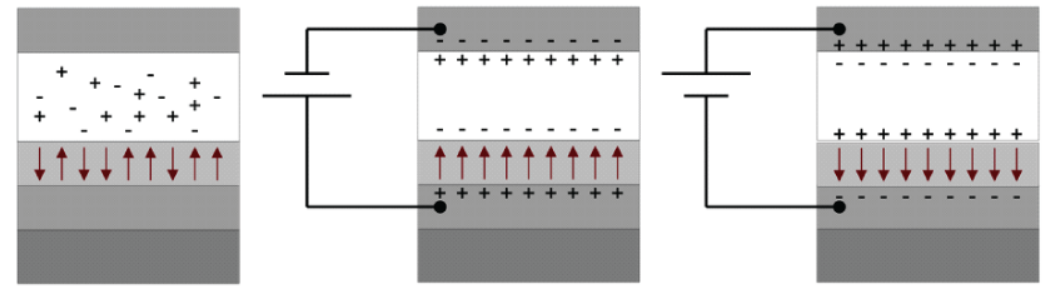

Figure 1. Ferroelectric cell release device and characteristics. (a) Experimental setup to characterize the ferroelectric switching of PVDF-TrFE in aqueous electrolyte. The inset shows the structure of PVDF-TrFE. (b) Typical current vs voltage characteristics. The scan started at $0 \mathrm{~V}$, proceeded to $18 \mathrm{~V}$, then to $-18 \mathrm{~V}$, then back to $0 \mathrm{~V}$. (c) Random dipole alignment before initial switching (left), after positive voltage switching (middle), and negative voltage switching (right). 

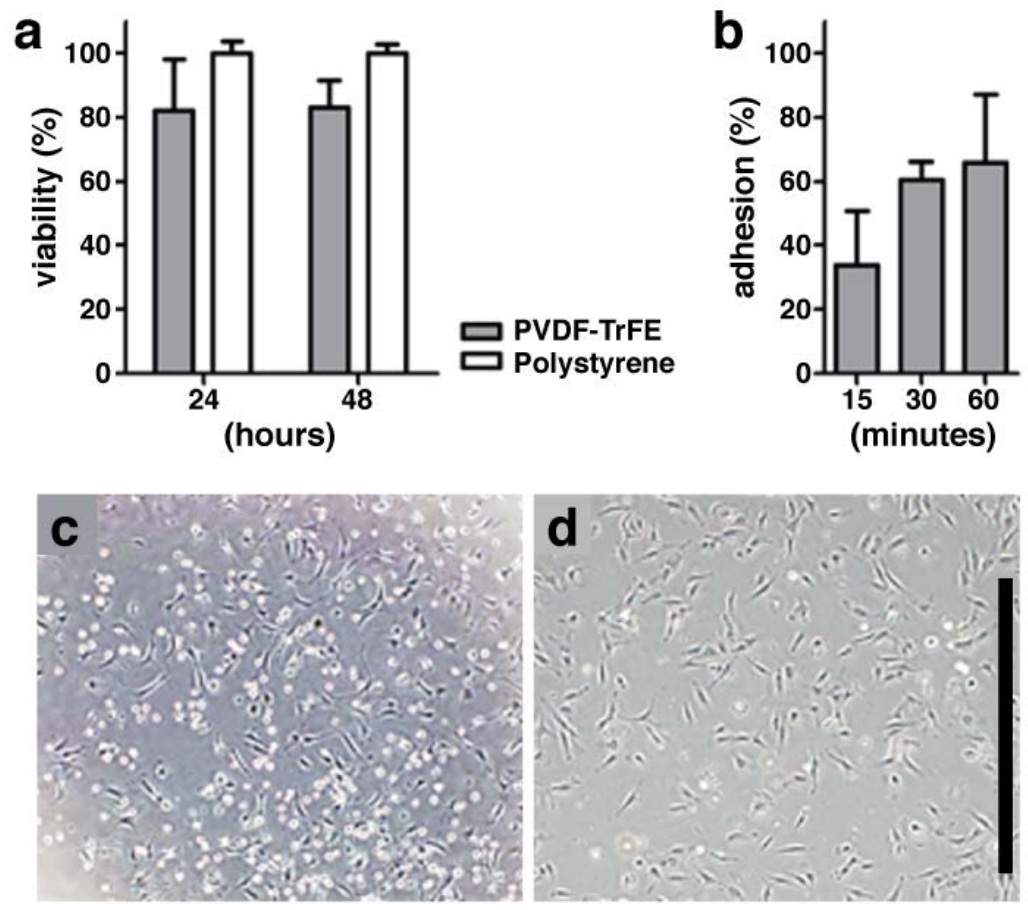

Figure 2. Fibroblast viability on and adhesion to unpolarized PVDF-TrFE. (a) Fibroblast viability on PVDFTrFE relative to fibroblasts on polystyrene control surface (mean \pm SEM, $n=6$ ). (b) Adhered fibroblasts on PVDF-TrFE after 15, 30 and 60 minutes as percentage of seeded cell amount (n=4). Primary fibroblasts on unpoled PVDF-TrFE (c) and polystyrene (d) 6 hours after seeding. Scale bar $=500 \mu \mathrm{m}$. 

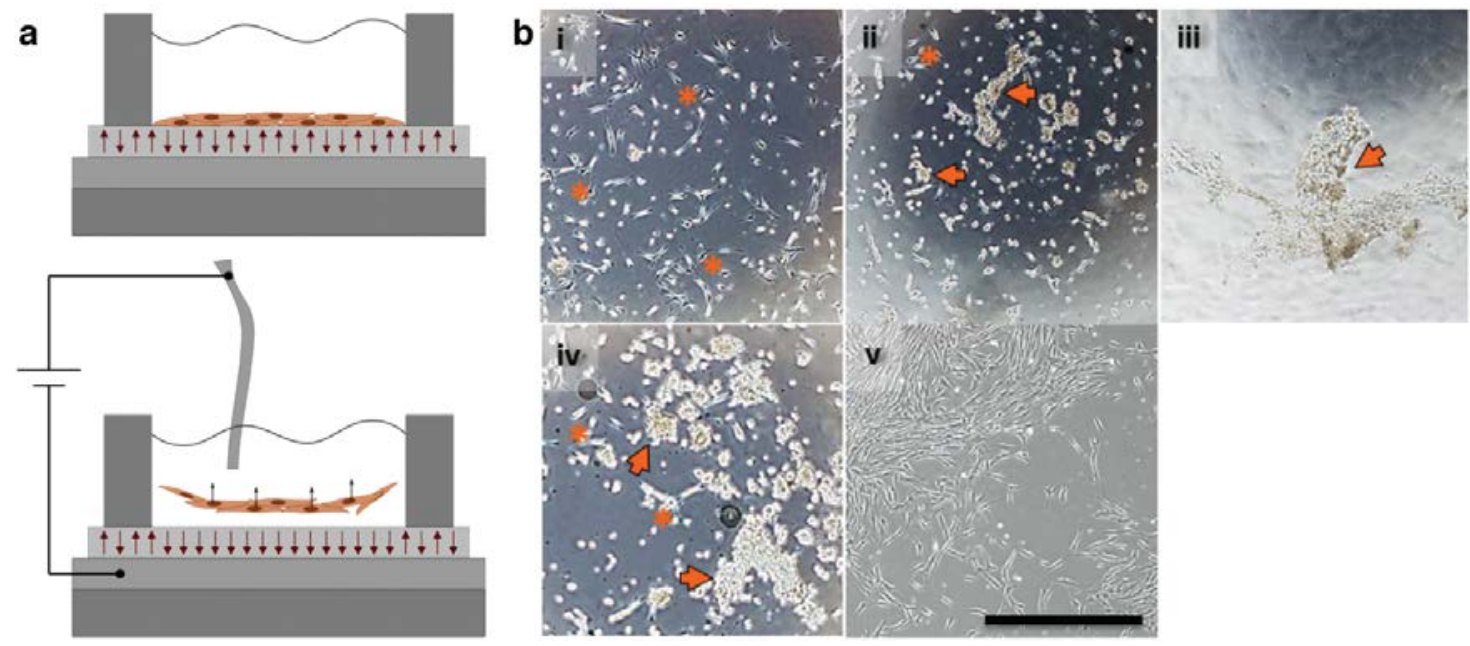

Figure 3. (a) Schematic illustration of the cell detachment experiment. Cells adhere and proliferate on the pristine unpolarized PVDF-TrFE surface (top). A sufficient voltage polarizes the ferroelectric film and the adhered cells detach from the negatively charged PVDF-TrFE surface (bottom). (b) Representative images of cell release from unpolarized surface cultured for 24 hours. (i) Initial morphology of fibroblasts on PVDFTrFE prior to detachment experiment. Fibroblasts exhibited normal, elongated morphology. (ii) and (iii) Detachment of fibroblasts after three successive $16 \mathrm{~ms}$ pulses of $-15 \mathrm{~V}$ (ITO as cathode). Asterisks (*) indicate attached fibroblasts, arrows indicate detached, floating cells. Note the detached cell sheet in (iii). (iv) Detachment of cells after fifteen successive $16 \mathrm{~ms}$ pulses of $-15 \mathrm{~V}$. The same experimental surface has been addressed and an increasing number of cells detach with increased number of pulses. (v) Morphology of collected cells reseeded on cell culture polystyrene after 72 hours of cell culture. Scale bar in all images is $1 \mathrm{~mm}$. 